\title{
COMPARISON OF THE LIGHTING CONDITION OF THE INTERIOR TO CREATE A 3D BACKGROUND IN VIRTUAL REALITY
}

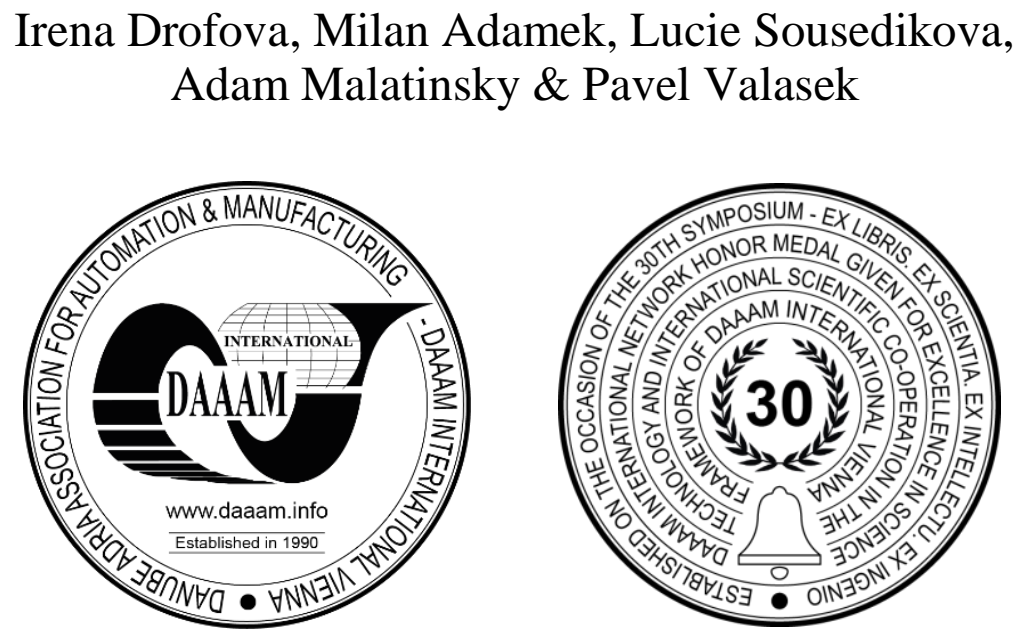

This Publication has to be referred as: Drofova, I[rena]; Adamek, M[ilan]; Sousedikova, L[ucie]; Malatinsky, A[dam] \& Valasek, P[avel] (2021). Comparison of the Lighting Condition of the Interior to Create a 3D Background in Virtual Reality, Proceedings of the 32nd DAAAM International Symposium, pp.0377-0383, B. Katalinic (Ed.), Published by DAAAM International, ISBN 978-3-902734-33-4, ISSN 1726-9679, Vienna, Austria

DOI: $10.2507 / 32$ nd.daaam.proceedings.055

\begin{abstract}
This article deals with capturing the image in the gallery's interior with a 3D camera in different lighting conditions. Currently, 3D backgrounds are a standard part of modelling and virtual reality. There are several ways to create a background, and there are various high-resolution 3D scanning devices. This study deals with capturing an image in the interior of a gallery with a 3D camera in different lighting conditions. The text lists the fundamental properties of light and lighting. The parameters and possibilities of capturing the image with a 3D camera are also described. The article further describes the indoor lighting conditions in the interior when using a 3D camera and compares the lighting conditions and their effect on the quality of the captured image and the goal of obtaining the best possible quality of the captured image.
\end{abstract}

Keywords: 3D camera; digital image; lighting condition; virtual reality; art gallery

\section{Introduction}

This article focuses on the preparation and acquisition of 3D image data for further processing in virtual reality. [1] Currently, 3D image data is used in many fields. There are many devices and techniques for capturing 3D images. Subsequent use of 3D image data then determines their further processing. Created 3D data and objects are used in many fields. They are used for technical documentation, simulations in industry, construction, architecture, design, and art. 3D models and 3D scanned images are also used in virtual reality. Image analysis and high-quality image data are essential, for example, in the field of medicine. The created 3D background as an interior simulation is essential for the degree of nesting in the virtual environment. Especially when simulating events or for training simulators. These are used in the army, rescue system units, or the police. 3D-created backgrounds based on actual data can thus significantly contribute to all the mentioned areas.

In this study, attention is focused on the field of art and works of art. In this field, great importance is placed on the image digital reproduction of materials and colours. Assessing the quality of the 3D image with respect to the lighting type is an essential step in image analysis, especially for the details captured in the picture. [2] 
The high image quality will also enable accurate image reproduction in 3D modelling and virtual reality. Digitization and its progressive development have also manifested themselves in this field. Online exhibitions and auctions are now a standard part of the art world and the art trade. High demands are placed on the reproduction of art and the quality of its depiction. Methods for identifying counterfeits are also changing in this area. There are many methods for distinguishing the original from the counterfeit. One of these methods is visual assessment. This method focuses on the artist's painting style, colours, scene, light. [3]

Modern computer vision techniques are currently used to obtain physical evidence of possible manipulations of a painting or the resolution of a forgery. [4] Therefore, it is essential to reproduce a work of art in a digital environment so that the reproduction of the work corresponds highly to the physical original. Significant differences in the observer's perception of a work of art can be caused by the type of lighting in the room and the illumination of the work of art. In this study, attention is focused on working with a 3D camera in the interior of the gallery. The image in the room was taken mainly in daylight.

\subsection{The light in the visible radiation}

The colour of the human eye perceives depends on the external environment and is variable in connection with its change. These are mainly lighting conditions and the environment, which significantly affect the resulting colour perception. In this chapter, attention is focused on the issue of Photometry, respectively methods that deal with visible radiation and in which the properties of the eye play an important role. Visible radiation is only a tiny part of the range of electromagnetic radiation and is a range of wavelengths of 380 to $760 \mathrm{~nm}$ that act on the retina of the human eye. Below are some photometric quantities, of which it is considered to be the central luminosity (I) in Photometry. [5]

The luminosity $(I)$ is, therefore, the primary photometric quantity. The unit of luminosity is a candela (cd). Luminosity expresses the ability of point sources to radiate in a given direction and to evoke visual perception.

Brightness / Luminance $(L)$ is a specific measure of the luminosity of a planar source. Brightness is the required ratio of the luminosity of the elementary surfaces of the sources $(d I)$ in the selected direction and the perpendicular projection of the surface in this direction $(\mathrm{dS} \cos \alpha)$ :

$$
L=\frac{d I}{d S \cos \alpha}
$$

Luminous flux $(\Phi)$ is a photometric quantity that expresses the ability of a given radiant flux to evoke visual perception. The unit is lumen $(\mathrm{lm})$ and is a derived unit. From approximately point sources with the omnidirectional luminosity of one candela $(I)$, the transmitter has a luminous flux $(\Phi)$ of the size of one lumen $(l m)$ up to the spatial angle of one steradian:

$$
\Phi=I \Omega
$$

Illumination $(E)$ is a specific measure of the luminous flux $(\Phi)$ on an illuminated surface. The unit of illumination is lux $(l x)$ :

$$
E=\frac{d \Phi}{d A}
$$

Image capture and its subsequent quality are affected by light. The image captured outdoors will be different from the image captured indoors. The resulting image will differ when shooting day and night. Other techniques used in photography also affect image quality. An object illuminated by artificial light or shadows suppressed by photo plates when shooting will be of a different quality. Complementary techniques also affect the shadows in the overall image. All these aspects and many more affect the final quality. All attributes affecting the resulting scene are adjustable during subsequent 3D modelling and light settings when creating a virtual environment.

\subsection{Type of the light sources}

Light sources are devices that emit visible radiation. These sources allow the creation of a particular lighting environment. This environment must comply with hygienic, environmental, and colorimetric standards. Standardized observation conditions are thus ensured. We divide electric light sources: [5]

- Heat sources: the electric current flowing through these sources heats the metal fiber. That excites the metal atoms. The spontaneous emission of photons produces thermal radiation with a continuous spectrum.

- Discharge sources: c electric discharge causes electrons and ions to collide with gas atoms or metal vapors. The energy of particles changes into optical radiation. Discharge lamps are often equipped with phosphors on the inner wall. These emit visible light when excited by UV radiation. The spectrum is linear or continuous with the spectral lines. 
- Laser radiation: monochromatic with high temporal and spatial coherence. This radiation is created by stimulated emission.

- Daylight: The source of daylight is the Sun. This light is significantly different from artificial light. The difference is mainly in the spectral composition and variability of radiation intensity. The spectrum is continuous with maximum intensity in the visible region.

\section{Type of $3 D$ camera for capture image}

There are currently many 3D scanning devices on the world market. [6] For example, laser 3D scanners are efficient and straightforward devices for instant 3D imaging with an extensive range in scanning angle, distance, and scanning speed. [7] Another option for capturing a 3D image is 3D cameras. There are also a large number of these devices with different applications. This text focuses on capturing the image in the gallery's interior using the 3D camera Insta360 Pro. [8]
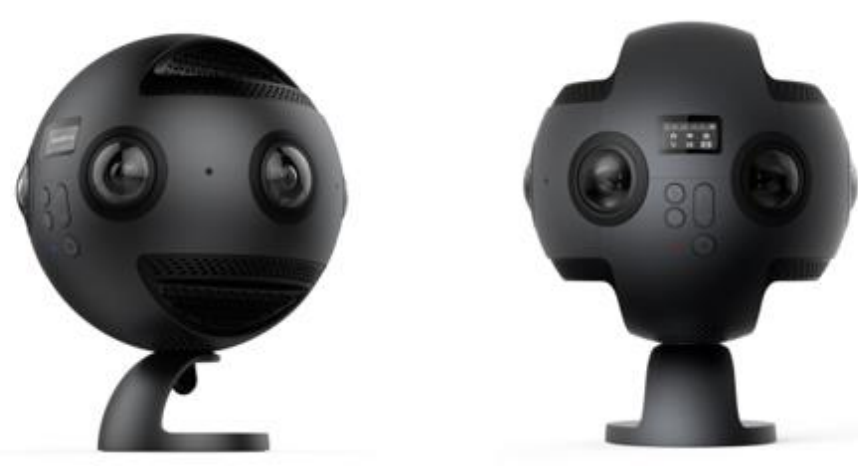

Fig. 1. The body of 3D camera Insta360 Pro [8]

This 3D and panoramic camera have 6 "fish eyes" with $200^{\circ} \mathrm{F} 2$ lenses around the circumference of the aluminum spherical body. That allows capturing high-quality $360^{\circ}$ images. The four microphones also provide good sound when recording video. In normal mode, the camera takes six still photos at a resolution of $4000 \mathrm{x} 3000$ px. These images can then be used to staple and subsequently process $8 \mathrm{~K}$ monoscopic and stereoscopic spherical images. The Insta360 stapling application is used for subsequent processing and stapling of the image into one unit. Insta360 Player is used to playing back stitched images. Insta360 Pro records data captured using an SD card (speed class V30 + necessary for video recording) and a USB SSD (USB3.0 interface). Insta360 Pro have static image modes:

- Normal mode captures six shots at 4000 x 3000 px, which are stapled into 8K monoscopic or stereoscopic images in post-processing.

- RAW mode is the primary mode for post-image processing for color calibration and other adjustments depending on image purpose. The file format is (.dng). Take six shots to staple $8 \mathrm{~K}$ monoscopic or stereoscopic spherical images sequentially.

- HDR mode is the mode is used to eliminate the difference in brightness. It takes three series of 6 shots at a resolution of 4000 x 3000 px, which are then used to staple an 8K highly dynamic spherical image. This mode does not allow automatic stapling directly in the camera.

- Burst mode is for a series of 10 with six images in resolution 4000 x 3000 px in one series. This mode does not allow automatic stapling of the captured image directly in the camera. Subsequent sewing again gives an $8 \mathrm{~K}$ image in monochromatic or stereoscopic.

- Static images can be captured by all six cameras manually by selecting one of the modes or remotely using the mobile app, ensuring convenient shooting in rugged terrain or confined conditions where there may be restrictions on manual handling and camera settings. Selected modes also enable real-time stapling. That will directly display the resulting panoramic image and can also display the image in virtual reality. The app needs to be reset every time use again. Likewise, when pairing mobile devices with wifi cameras.

The application for controlling and setting the 3D camera allows displaying the captured image directly in virtual reality. In this way, the camera also allows previewing the image before capturing it. That not only allows to adjust the lighting and scene before shooting. In the application and the display in virtual reality, it is possible to see the image's quality and the fidelity of the reproduction of works of art. These can be seen in digital form and partly affect the quality of their digitization. Colour vision and colorimetry are essential for subsequent image processing in digital form. [9] The colours transferred between colour spaces [10] and the definition of colours in the digital environment will allow a highquality reproduction of the original work of art. The following chapter describes the process of capturing the gallery interior in different lighting. 


\section{3D scanning by $3 D$ camera in the interior}

The Insta360 Pro 3D camera has 6 lenses around the perimeter of the body. These are $200^{\circ} \mathrm{F} 2$ "fish eye" lenses. These types of objects are not fully suitable for 3D modelling by photogrammetry. For example, they are not recommended for processing in Agisoft Metashape Professional software. [11] Editing a 3D model would be very challenging. The model developed in this software is shown in Figure 2.
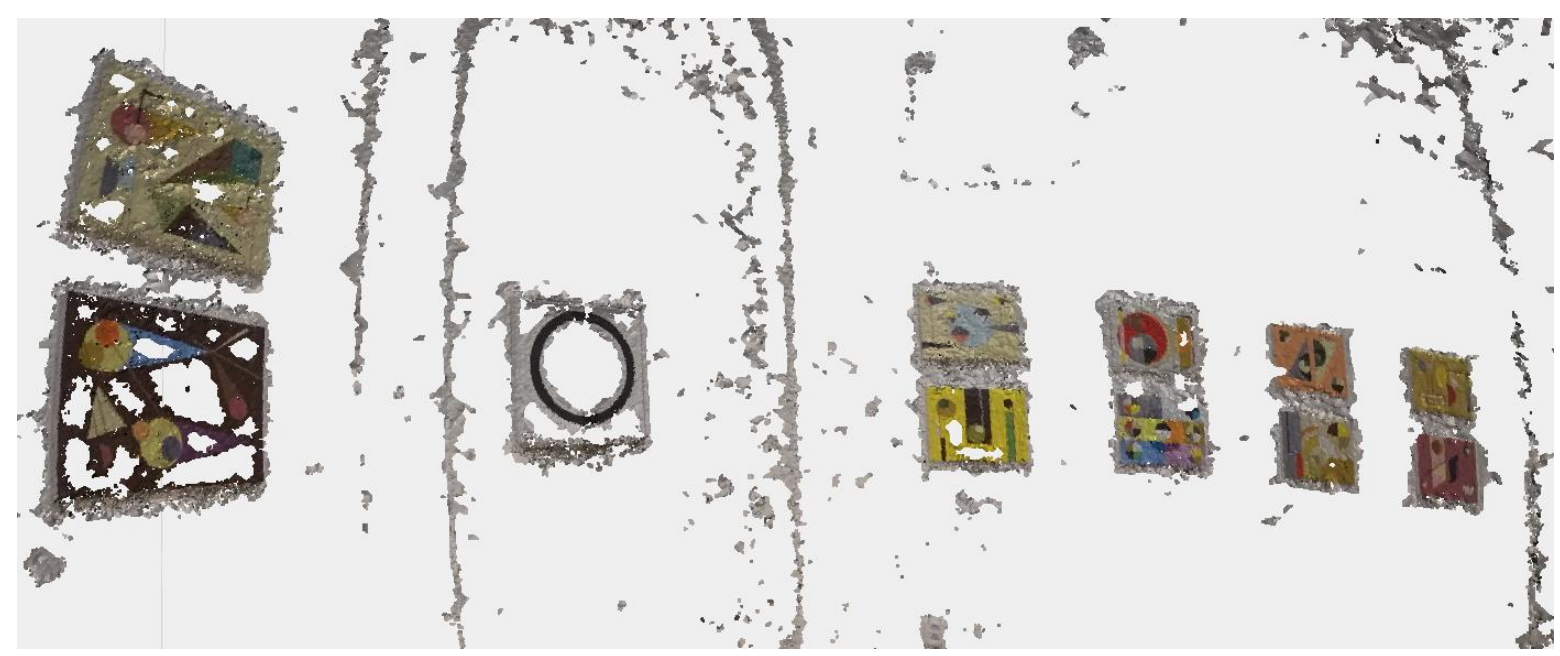

Fig. 2. The 3D model gallery's interior by software Agisoft Metashape Professional

\subsection{Capturing an images and interior light}

Indoor image capture has its specifics. In particular, lighting and scanning devices have a significant influence on the quality of the acquired image. The lighting in the room can be daylight or artificial. Both types of lighting may also be required. In art galleries, it is also possible to illuminate individual exhibits separately. This lighting is chosen to underline the artwork. As a rule, however, works of art are very well observed in daylight. The lighting of works of art usually chooses to be as close as possible to daylight. For the colour vision of the human eye, the colours of the works of art are better seen in interior lighting close to daylight. In this study, the interior of the gallery in a historic building was chosen. Daylight is natural in this interior. Artificial lighting is used to dim the room. That is a classic interior lamp with discharge sources. In the subsequent text, both types of lighting are compared to the display of works of art in the gallery's interior.
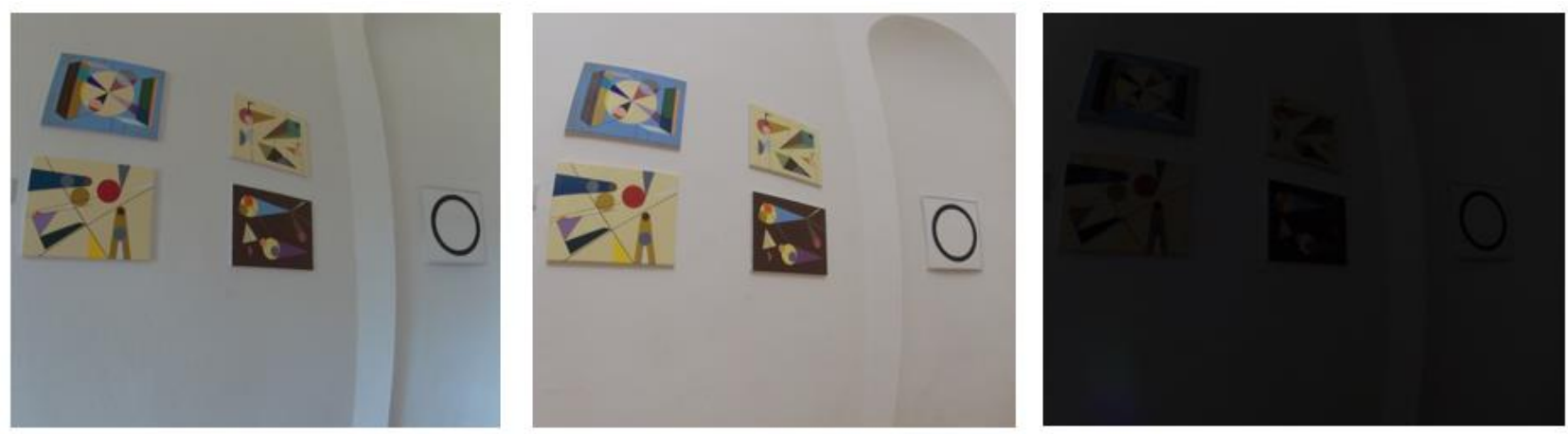

Fig. 3. Picture captured in the gallery's interior: daylight / discharge sources lamp / without light

Figure 3 shows a comparison of the image captured during three different lighting conditions. The first image shows the quality of the image captured in daylight, the second figures shows quality of the image in discharge sources lighting and the third figure shows image in the dark interior. The room has one large door towards the terrace. It was sunny when the picture was taken indoors. However, sunlight did not fall into the room. It can be seen that the captured image is directed in blue tones in the nature day illumination. This image will need to be subsequently adjusted to minimize the blue tone and the noise created in the shadows of the corner in the room. That image captured in discharge lighting lamp in the interior have more high quality. There is no blue tone in the image. At first glance, however, it is clear that the image is tinted slightly to a red hue. Also, this image and its quality can be improved by further editing in the graphics software. In the case of an image captured without lighting, i.e. in the dark, it is clear that the image quality is very low. Works of art can only be minimally recognized in the image. The image captured in this way is not suitable for further processing. 


\subsection{Types and possibilities of capturing the image with a $3 D$ camera}

The previous chapter lists the formats that can capture an image with the Insta360 Pro 3D camera. This scanning device has many uses. The camera can take static photos with this 3D camera. It will create a 3D image with these photos and their stitching. The camera can also take panoramic photos. For 3D modelling, it is appropriate to use the 3D format. These are three ways to create an environment or background in 3D models and a virtual environment. Last but not least, the camera is also suitable for recording high-quality video and for a live broadcast or stream. The following figures show some of the above types of captured images. The images were obtained in the gallery's interior with a discharge light source.
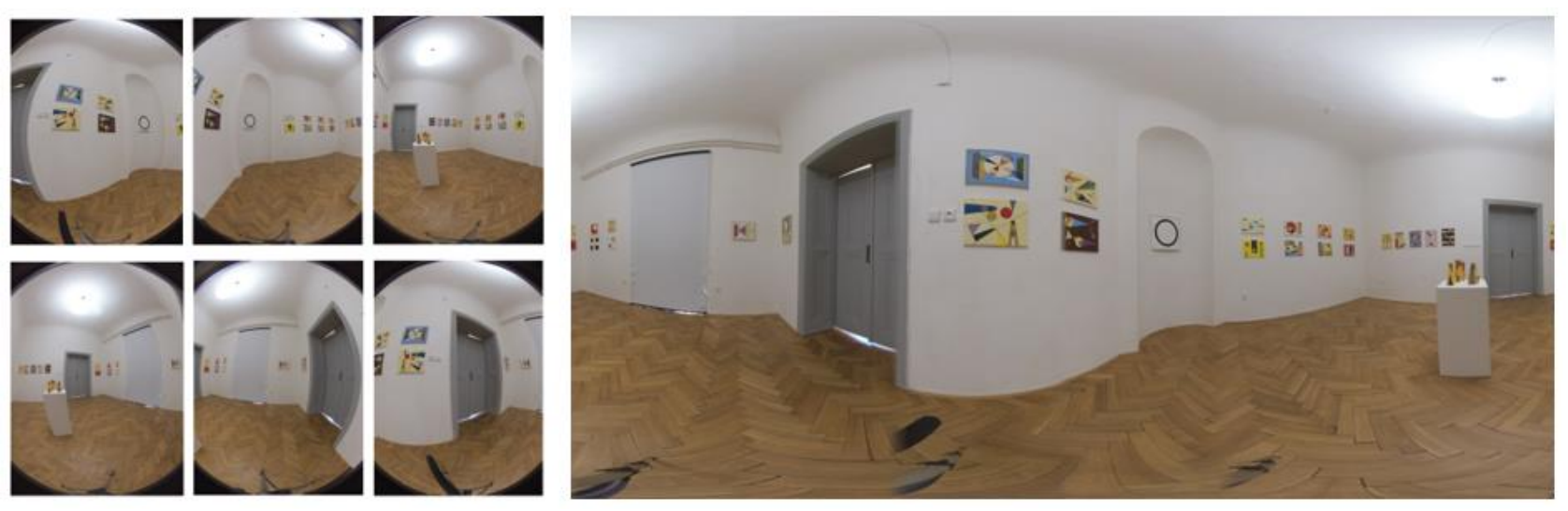

Fig. 4. The type of six static photos $\left(200^{\circ} \mathrm{F} 2\right.$ "fish eye" lenses) and their stitching
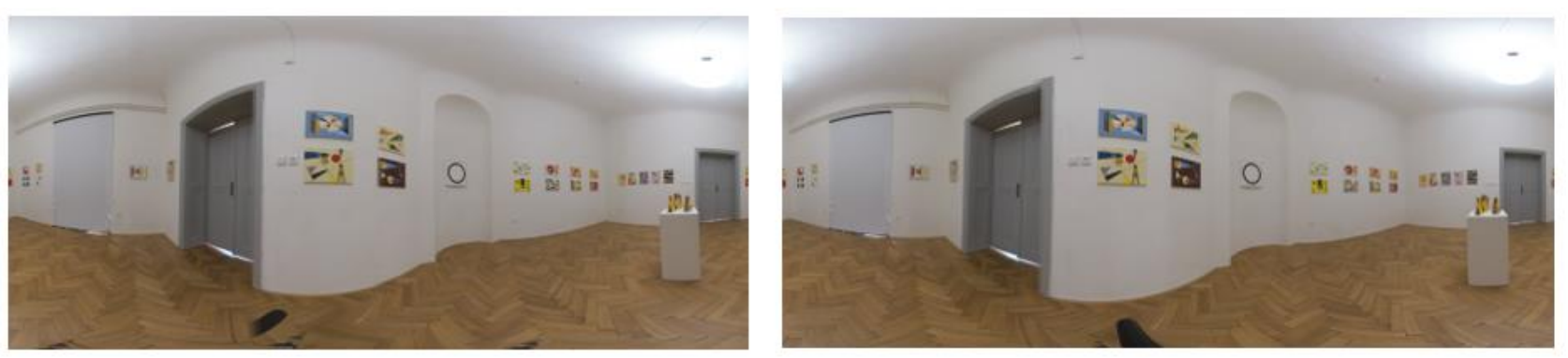

Fig. 5. The type of the panoramatic photo: the left and the right panoramatic photo

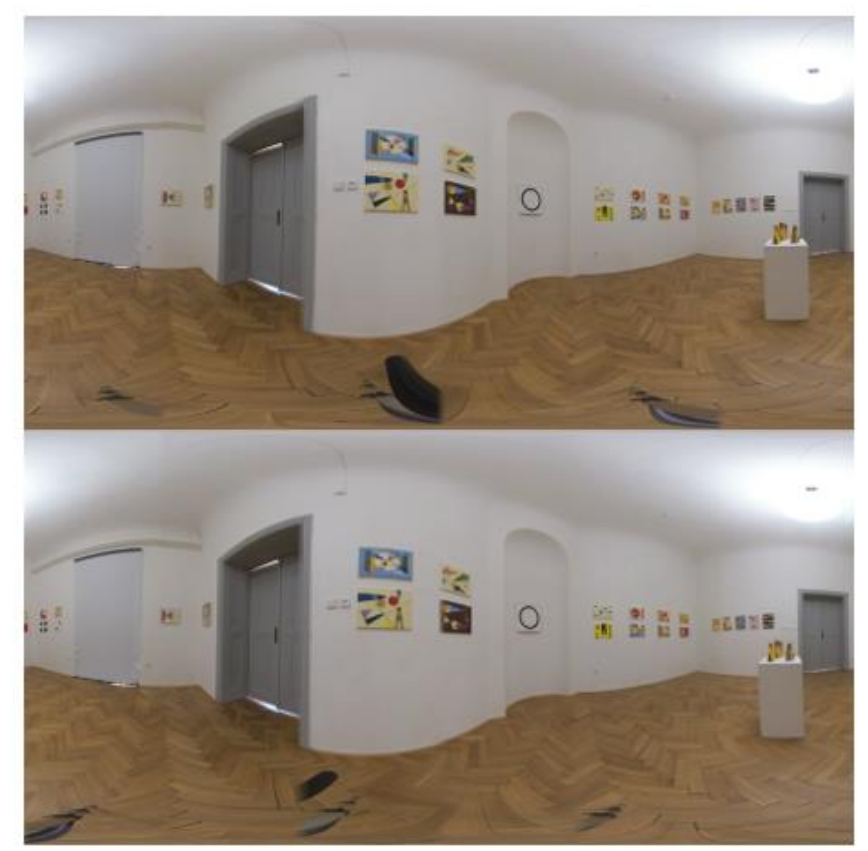

Fig. 6. The type of 3D photo 
In this particular case, the Insta360 Pro's 3D camera captured the image using a natural interior environment with one discharge lamp in the middle of the room. The room also has a large wing glass door facing west with access to the terrace. The paintings were taken in August 2019 at the time of year with the highest intensity of daylight. The aim was to determine what image quality would be captured by this particular device and to what extent it would be necessary to modify the captured image in graphics software. Therefore, no photographic accessories have been used to improve image quality and suppress side effects. These accessories and tools would increase the quality of the scanned image.

\section{Conclusion}

Many methods are currently used in modern computer graphics to create backgrounds, material structures, and virtual environments. In many fields, it is desirable to create a real digital image. In this study, attention was focused on the field of art. In the modern digital world, there is a need for the high-quality digital reproduction of works of art for online exhibitions, online art auctions, or virtual reality art.

This work aimed to find out to what extent a quality image of the interior can be obtained so that this image does not have to be complicatedly modified. Emphasis was placed on the possibility of capturing an image in different lighting of a room with works of art. A high-quality 3D camera captured this interior with several modes for further use. This article focuses on the possibilities of creating a 3D image for further use in the field of 3D graphics and virtual reality. The image was taken on a sunny day, and the natural daylight sufficiently illuminated the entire interior with the images. Another capture of the room was under artificial lighting. A discharge light source illuminated the space. In addition, an image of the interior with minimal lighting was taken for further assessment

The assumption of the high quality of the captured image in daylight has not been confirmed. The image thus obtained showed a high degree of blue tone and noise in the shadows. In an environment with artificial lighting, a worse red tone appeared but minimal. These phenomena can be easily eliminated in computer graphics and thus obtain a higher quality digital image, especially for quality reproduction of colours used to create works of art in the interior. For these needs, it is still necessary to define the colours in the colour spaces so that the colours tones are not fundamentally changed during further adjustments. Both types of scanned images could be further detected after adjustments. They may be appropriate for an initial view in virtual reality, where you can continue working with light and its properties. In the case of creating a real virtual gallery, this would have to be necessary. On the contrary, a captured image in a dark environment cannot sufficiently perform works of art. In this case, it would be appropriate to use external lighting and other photographic accessories, especially for capturing the image at night.

All three image capture methods in Chapter 3 could be used to create backgrounds in 3D modelling and virtual reality. Definition of colours and quality processing of works of art would precede further processing. In many modelling software and virtual reality programs, working with light is very important. That would also make it possible to suppress some of the undesirable phenomena and, on the contrary, to add others, which would make the space more pleasant for observers.

The 3D camera used in this study is suitable for both interior and exterior. No photographic accessories or image enhancement devices were used to capture the image indoors. The aim was to find out what it would look like in the live stream in an actual image into virtual reality. Subtle tonal variations could partially limit the calibration of colours on the virtual reality headset, however, not in the case of a captured image without lighting, which does not show the exhibited works of art insufficient quality and are displayed only minimally. An image captured in daylight and artificial lighting would be suitable for the live broadcast from the opening of exhibitions by the artist. On this occasion, the artist presents his works and adds commentary to them. This way of transmitting the captured image will be further research into the opening of another art exhibition.

The pictures were taken in the daytime, and artificial lighting shows incomparable colour tones. This reduced image quality can increase image editing and correction in graphics software. Use the process to build seamlessly on this study. The digital image will increasingly be used as a background in the created model of the virtual gallery with an exhibition of the authors of the created more miniature sculptures in the same artistic style.

\section{Acknowledgments}

This research was based on the support of the author of the paintings, Mr. Michal Pasma, Vaclav Chad Gallery in Zlin, and of the International Grand Agency of Thomas Bata University in Zlin, IGA/CebiaTech/2021/004, and the Department of Security Engineering, Faculty of Applied Informatics.

\section{References}

[1] S. Martirosov \& P. Kopecek, (2017). Virtual Reality and its Influence on Training and Education - Literature Review, Proceedings of the 28th DAAAM International Symposium, pp.0708- 0717, B. Katalinic (Ed.), Published by DAAAM International, ISBN 978-3-902734-11-2, ISSN 1726-9679, Vienna, Austria DOI: 10.2507/28th.daaam.proceedings. 100

[2] V. Huskova, V. Adamova, and K. Boc, (2021) Use of 3D Screening Technology Traks in the Process Criminal Identification (SK), Young Science Conference, Tomas Bata University in Zlin, Facilty of Applied Informatics, Trilobit, [online], http://trilobit.fai.utb.cz/Data/Articles/PDF/8dcada30-4b45-403b-a048-2c5d1026855a.pdf 
[3] E. Gultebpe, E. Thomas, and M. M. Conturo, (2018) Predicting and grouping digitized paintings by style using unsupervised feature learning, Journal of Cultural Heritage., vol. 31, 13-23., ISSN 1296-2074., DOI:10.1016/j.culher.2017.11.008

[4] A. Pelagotti, A. Piva, F. Uccheddu, D. Shullani, M. F. Alberghina, S. Schiavone, E. Massa and C. M. Menchetti, (2020) Forensic Imaging for Art Diagnostics. What Evidence Should. We Trust?, IOP Publishing, IOP Conf. Series: Materials Science and Engineerin, 012076, 949 doi:10.1088/1757-899X/949/1/012076

[5] M. Dohnal, (2007) Physical Bases of Image Reproduction, University of Pardubice , ISBN: 978-80-7194-945-9

[6] Z. Stojkic, E. Culjak \& L. Saravanja, (2020), 3D Measurement - Comparison of CMM and 3D Scanner, Proceedings of the 31st DAAAM International Symposium, pp.0780-0787, B. Katalinic (Ed.), Published by DAAAM International, ISBN 978-3-902734-29-7, ISSN 1726-9679, Vienna, Austria. DOI: 10.2507/31st.daaam.proceedings. 108

[7] Gefos ČR Leica BLK360 (2021). [online], https://www.gefos-leica.cz/o-produktech/3d-laserove-skenovani/3dlaserove-skenery/blk360

[8] Insta 360 Pro, (2021), [online], https://www.insta360.com/product/insta360-pro/

[9] M. Dohnal, (2019) Color vision. Colorimetry, University of Pardubice, ISBN: 978-80-7560-246-6

[10] P. Ehkan, S. V. Siew, F. F. Zakaria, M. N. M. Warip and M. Z. Ilyas, (2020) Comparative Study of Parallelismand Pipelining of RGB to HSL Colour Space Conversion Architectureon FPGA, IOP Conf. Series: Materials Science and Engineering., DOI:10.1088/1757-899X/767/1/012054

[11] Agisoft (2021). [online], https://www.agisoft.com/features/professional-edition/ 